\title{
Controlling Light with Light in a Plasmonic Nanooptomechanical Metamaterial
}

\author{
Jun-Yu Ou ${ }^{1}$, Eric Plum ${ }^{1}$, Jianfa Zhang ${ }^{1}$, and Nikolay I. Zheludev ${ }^{1,2}$ \\ ${ }^{1}$ Optoelectronics Research Centre and Centre for Photonic Metamaterials, University of Southampton, SO17 1BJ, UK \\ ${ }^{2}$ Centre for Disruptive Photonic Technologies, Nanyang Technological University, Singapore 637378, Singapore \\ erp@orc.soton.ac.uk, niz@orc.soton.ac.uk
}

\begin{abstract}
We demonstrate metamaterial with a cubic optical nonlinearity that is ten orders of magnitude greater than the reference nonlinearity of $\mathrm{CS}_{2}$. The nonlinearity has optomechanical nature and is underpinned by light-induced electromagnetic near-field interactions.

OCIS codes: (160.3918) Metamaterials; (120.4880) Optomechanics.
\end{abstract}

\section{Introduction}

We experimentally demonstrate for the first time that light can control light in a plasmonic metamaterial using nonlinearity of nanooptomechanical nature. At the nanoscale light-driven electromagnetic near-field interactions and thermo-optical effects can overcame elastic forces: light intensities of only a few $\mu \mathrm{W} / \mu \mathrm{m}^{2}$ can reconfigure the metamaterial array of plasmonic metamolecules fabricated on a flexible substrate leading to a significant change of its optical properties. This new type of nonlinearity has a resonant character and can provide light-by-light modulation with $\mathrm{MHz}$ bandwidth.
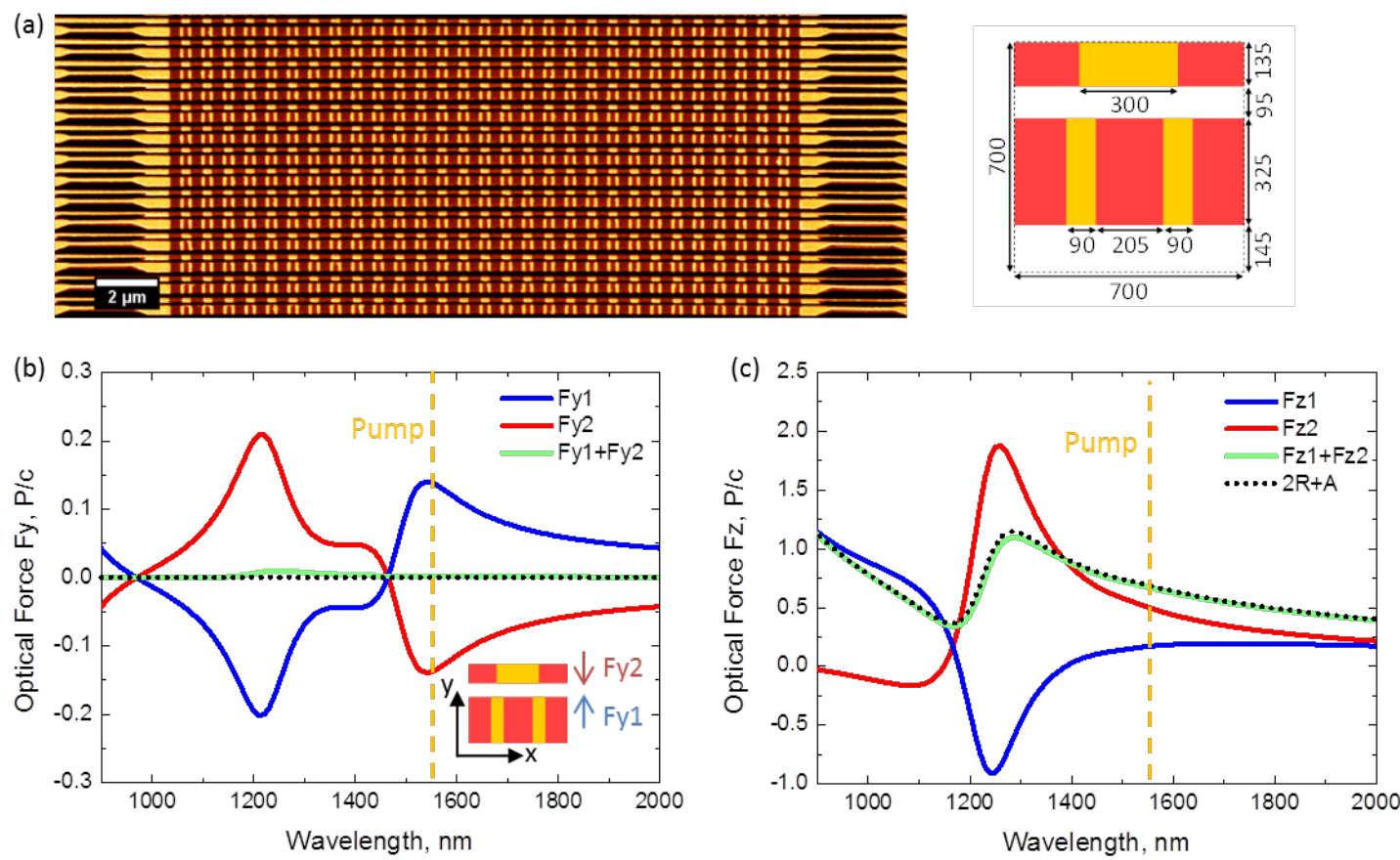

Fig. 1. (a) Scanning electron microscope image of an optically reconfigurable metamaterial nanostructure consisting of gold (yellow) plasmonic resonators supported by free-standing silicon nitride bridges (red). The inset shows an individual unit cell. (b,c) Simulated optical forces between the bridge segments of an individual $700 \times 700 \mathrm{~nm}^{2}$ unit cell acting (b) in the metamaterial plane and (c) along the propagation direction normal to the metamaterial plane. Dashed lines indicate the $1550 \mathrm{~nm}$ optical pump wavelength.

\section{Results}

The optomechanical photonic metamaterial studied here is based on a П-shaped resonator design known for exhibiting plasmon-induced transparency, see Fig. 1a. In order to allow mechanical deformation of the plasmonic $\Pi$ meta-molecules, the horizontal and vertical bars have been supported by different flexible dielectric bridges. The nanostructures were fabricated by focused ion beam milling from a $50 \mathrm{~nm}$ thick silicon nitride membrane covered with a $50 \mathrm{~nm}$ thick thermally evaporated layer of gold. 
Maxwell stress tensor calculations reveal optical forces acting on the П-resonators around their $1240 \mathrm{~nm}$ absorption resonance, see Fig. 1b,c. As the normally incident photons only carry momentum along the $z$-direction, there cannot be any net in-plane optical forces on the unit cell, $F_{y 1}+F_{y 2}=0$, and the net force along the $z$-direction must be given by the momentum transfer associated with absorption and reflection, $F_{z 1}+F_{z 2}=(A+2 R) P / c$, where $A$ is absorption, $R$ is reflection, $P$ is the incident power per unit cell and $c$ is the speed of light in vacuum. In close agreement with these relationships, our simulations show substantial relative optical forces acting on different components of the unit cell. The relative optical forces $F_{2}-F_{1}$ between the unit cell's bridge segments reach about 0.4 $\mathrm{P} / \mathrm{c}$ along y and about $3 \mathrm{P} / \mathrm{c}$ along $z$.

In order to optomechanically modulate the metamaterial's optical properties, we pumped the nanostructure with a modulated laser beam at $1550 \mathrm{~nm}$, where simulations predict significant relative forces on the bridges along $y$. The modulation of the metamaterial's transmission was probed at $1310 \mathrm{~nm}$ and detected using a lock-in amplifier.

At modulation frequencies of $10 \mathrm{~s}$ of $\mathrm{kHz}$, the optical pump leads to pronounced modulation of the structure's transmission characteristics at the probe wavelength, see Fig. 2. For a pump power of $0.66 \mathrm{~mW}$ (peak intensity $I$ $=250 \mathrm{~W} / \mathrm{cm}^{2}$ ) a modulation amplitude on the order of $1 \%$ is detected at $25 \mathrm{kHz}$ modulation. However, the modulation amplitude drops rapidly with increasing modulation frequency, approaching the detection limit of about $0.0025 \%$ at a few $100 \mathrm{~s}$ of $\mathrm{kHz}$. The rapid decay in the modulation amplitude at $\mathrm{kHz}$ frequencies points towards a thermal origin of the effect, which is confirmed by a detailed analysis. Here, optical heating leads to differential thermal expansion of the gold and silicon nitride layers, resulting in deformation of the nanostructure.

Importantly, optical modulation of the metamaterial transmission is also seen at the nanostructure's mechanical resonances, which occur at $\mathrm{MHz}$ frequencies and have been identified as the fundamental out-of-plane and in-plane oscillation modes of the bridge beams, see Fig. 2a. Here, the oscillation period of about $1 \mu$ s is much shorter than the bridge cooling timescale of $10 \mathrm{~s}$ of $\mu$ s suggesting a non-thermal excitation mechanism. In particular, the $1 \mathrm{MHz}$ and 1.4 MHz mechanical resonances correspond to in-plane oscillation, which cannot be excited directly by thermal expansion of the gold and silicon nitride layers. Detailed calculations show that the observed light-by-light modulation can be explained by resonantly enhanced bridge deformation by near-field optical forces, see Fig. 1c.

As the transmission modulation depends linearly on the pump intensity, see Fig. 2b, it can be described by the first nonlinear absorption coefficient $\beta$. Assuming that the nonlinear transmission change $\Delta T$ results from nonlinear absorption, $\beta \sim \Delta T /(I t)$, where $I$ is the intensity and $t$ is the metamaterial thickness. In the low frequency limit $\beta \sim 10^{-2}$ $\mathrm{m} / \mathrm{W}$, which exceeds the nonlinearity of the classic nonlinear reference medium of $\mathrm{CS}_{2}$ by 10 orders of magnitude.
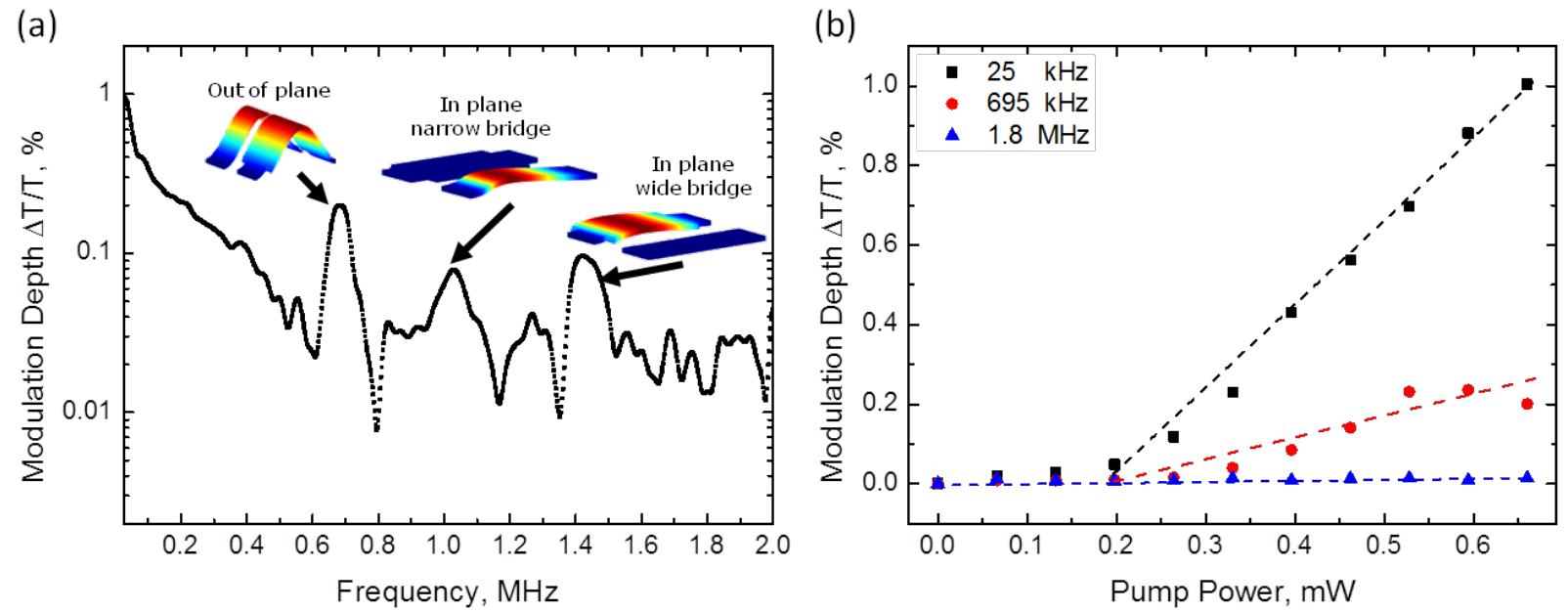

Fig. 2. Observation of light-by-light modulation. (a) Modulation depth as a function of modulation frequency for a pump power of $0.66 \mathrm{~mW}$ on a logarithmic scale, where simulations of the mechanical eigenmodes are shown as insets. (b) Modulation depth as a function of pump power at selected modulation frequencies.

\section{Summary}

In summary, we demonstrate a novel class of optomechanical metamaterials exhibiting exceptionally large optical nonlinearities resulting from rearranging the metamaterial nanostructure with light. We provide evidence of two mechanisms of optomechanical deformation, (i) optical heating leading to deformation resulting from differential thermal expansion of the constituent materials and (ii) nearfield optical forces between plasmonic nanostructures. 